\title{
RIESZ'S FUNCTIONS AND CARLESON INEQUALITIES
}

\section{TAKAHIKO NAKAZI}

Abstract. Let $\mu$ be a finite positive Borel measure on the open unit disc $D$ and $H$ a set of all analytic functions on $D$. For each $a$ in $D$, put

$$
r(\mu, a)=\sup |f(a)|^{2}
$$

where $f \in H$ and $\int_{D}|f|^{2} d \mu \leqslant 1$. Unless the support set of $\mu$ is a finite set, $\int_{D} r(\mu, a) d \mu(a)=$ $\infty$. However

$$
\sup _{z \in D} \int_{D_{t}(z)} r(\mu, a) d \mu(a)<\infty
$$

may happen where $D_{t}(z)$ denotes the Bergman disc in $D$. We study when this is possible. When $v$ is a discrete measure such that $d v=\sum_{a \in A} s(\mu, a) \delta_{a}$,

$$
\sup _{z \in D} \int_{D_{t}(z)} r(\mu, a) d v(a)=\sup _{z \in D} \sum_{a \in A \cap D_{t}(z)} 1
$$

Under some condition on $\mu$, we show that $\sup _{z \in D} \int_{D_{t}(z)} r(\mu, a) d v(a)<\infty$ for a finite positive Borel measure $v$ on $D$ if and only if $(v, \mu)$-Carleson inequality is valid.

Mathematics subject classification (1991): 46E15, 30A98, 47B99. sequence.

Key words and phrases: Bergman space, weight, Riesz's function, Carleson inequality, interpolation

\section{REFERENCES}

[1] T. NAKAZI AND M. YAMADA, $\left(A_{2}\right)$-conditions and Carleson inequalities in Bergman spaces, Pacific J. Math., 173 (1996), 151-171.

[2] T. NAKAZI AND M. YAMADA, Riesz's functions in weighted Hardy and Bergman spaces, Canad. J. Math., 48 (1996), 930-945.

[3] W. Rudin, Real And Complex Analysis, Third Edition, McGraw-Hill, Inc., New York, Tronto and London, 1987.

[4] K. ZHU, Evaluation operators on the Bergman space, Math. Proc. Cambridge. Philos. Soc., 117 (1995), 513-523.

[5] K.ZHu, Operator Theory In Function Spaces, Pure and Applied Mathematics, Marcel Dekker, Inc.,New York and Basel, 1990. 